DrAFT VERSION JULY 7, 2021

Preprint typeset using $\mathrm{IAT}_{\mathrm{E}} \mathrm{X}$ style emulateapj v. 5/2/11

\title{
EFFECTS OF SPOT SIZE ON NEUTRON-STAR RADIUS MEASUREMENTS FROM PULSE PROFILES
}

\author{
Michi Bauböck, Dimitrios Psaltis, and Feryal Özel \\ Astronomy Department, University of Arizona, 933 N. Cherry Ave., Tucson, AZ 85721, USA \\ Draft version July 7, 2021
}

\begin{abstract}
We calculate the effects of spot size on pulse profiles of moderately rotating neutron stars. Specifically, we quantify the bias introduced in radius measurements from the common assumption that spots are infinitesimally small. We find that this assumption is reasonable for spots smaller than $10^{\circ}-18^{\circ}$ and leads to errors that are $\leq 10 \%$ in the radius measurement, depending on the location of the spot and the inclination of the observer. We consider the implications of our results for neutron star radius measurements with the upcoming and planned X-ray missions NICER and LOFT. We calculate the expected spot size for different classes of sources and investigate the circumstances under which the assumption of a small spot is justified.

Subject headings: stars: neutron — relativity — gravitation
\end{abstract}

\section{INTRODUCTION}

Neutron stars provide a natural testbed for a variety of interesting physical phenomena. At their cores, neutron stars contain matter at densities and pressures unachievable in the laboratory setting. By measuring their properties, it is possible to constrain the unknown equation of state of cold dense matter. In particular, simultaneous mass and radius measurements with better than ten percent accuracy are needed to reach this goal (e.g., Lindblom 1992; Lattimer \& Prakash 2001; Read et al. 2009; Özel \& Psaltis 2009). Moreover, such measurements can further be used for testing the limits of General Relativity and alternate theories of gravity (e.g., Psaltis 2008).

One promising avenue for simultaneous mass and radius measurements is through the observations of pulse profiles originating from temperature anisotropies on the stellar surface. These anisotropies appear on a variety of isolated and accreting sources. In isolated pulsars, magnetic return currents heat the region around the magnetic pole, creating a local hotspot (e.g., Ruderman \& Sutherland 1975; Arons 1981; Harding \& Muslimov 2001, 2002; Bai \& Spitkovsky 2010a, b). In accreting neutron stars, the hotspots arise from matter funneled onto a small region of the surface (see Frank et al. 2002 and references therein). Alternatively, in X-ray bursters, the thermonuclear burning is thought to ignite at one spatially localized point in the accreted layer and takes many spin periods to spread across the stellar surface, creating a short-lived temperature anisotropy (e.g., Schoelkopf \& Kelley 1991; Strohmayer 1992, Strohmayer et al. 1996; Bildsten 1995). In all of these cases, the amplitude and shape of the pulse profile encodes information about the size and location of the spot as well as the properties of the neutron star.

In order to extract the mass and radius from the pulse profile shape, accurate theoretical models are needed that take account gravitational lensing, Doppler shifts, and time delays from the neutron-star surface. Several studies to date have focused on modeling pulse profiles, including a variety of relativistic effects. The sim-

mbaubock@email.arizona.edu plest approximation is to model the star as a sphere in the Schwarzschild metric and separately calculate the Doppler shift and aberration due to the rotation of the stellar surface (e.g., Miller \& Lamb 1998; Muno et al. 2002, 2003; Lamb et al. 2009a, b; Lo et al. 2013). Poutanen \& Beloborodov (2006, see also Bogdanov et al. 2007) used this model to find analytic approximations for the photon lensing and time delays and constructed analytic lightcurves for neutron-star pulse profiles.

The Schwarzschild metric describes a spherical nonspinning mass and is, therefore, an appropriate limit for slowly spinning neutron stars. For faster spins, however, effects at increasing order of spin frequency become important. At first order in spin frequency, frame dragging affects the spacetime around a spinning star, which is described by the Kerr metric. Braje et al. (2000) modeled pulse profiles in the Kerr metric and found that the distortion of the profile due to these effects is at the $1 \%$ level. At second order in spin frequency, the neutron star becomes oblate in shape and acquires a significant quadrupole moment. Cadeau et al. (2007, see also Morsink et al. 2007) found that the oblateness significantly alters the pulse profile, causing the spot to be visible at inclinations where it would be eclipsed by a spherical star. Psaltis \& Özel (2014) additionally included the effect of an appropriate quadrupole moment by using the Hartle-Thorne metric, which is formally correct up to second order in spin frequency. Even higher orders in spin frequency can introduce additional effects to the spacetime. Cadeau et al. (2007) modeled lightcurves for rapidly spinning neutron stars by calculating the metric numerically. They found that corrections from higherorder terms are negligible for the spin frequencies of known neutron stars.

The problem of modeling pulse profiles and, in turn, inferring neutron-star properties from pulse profiles, is difficult because of the large number of parameters needed. In principle, for any neutron-star model, the pulse profile depends on the properties of the neutron star as well as the size, shape, and position of the hotspot on the stellar surface. In order to simplify the models and reduce the parameter space, pulse profiles are often calculated un- 

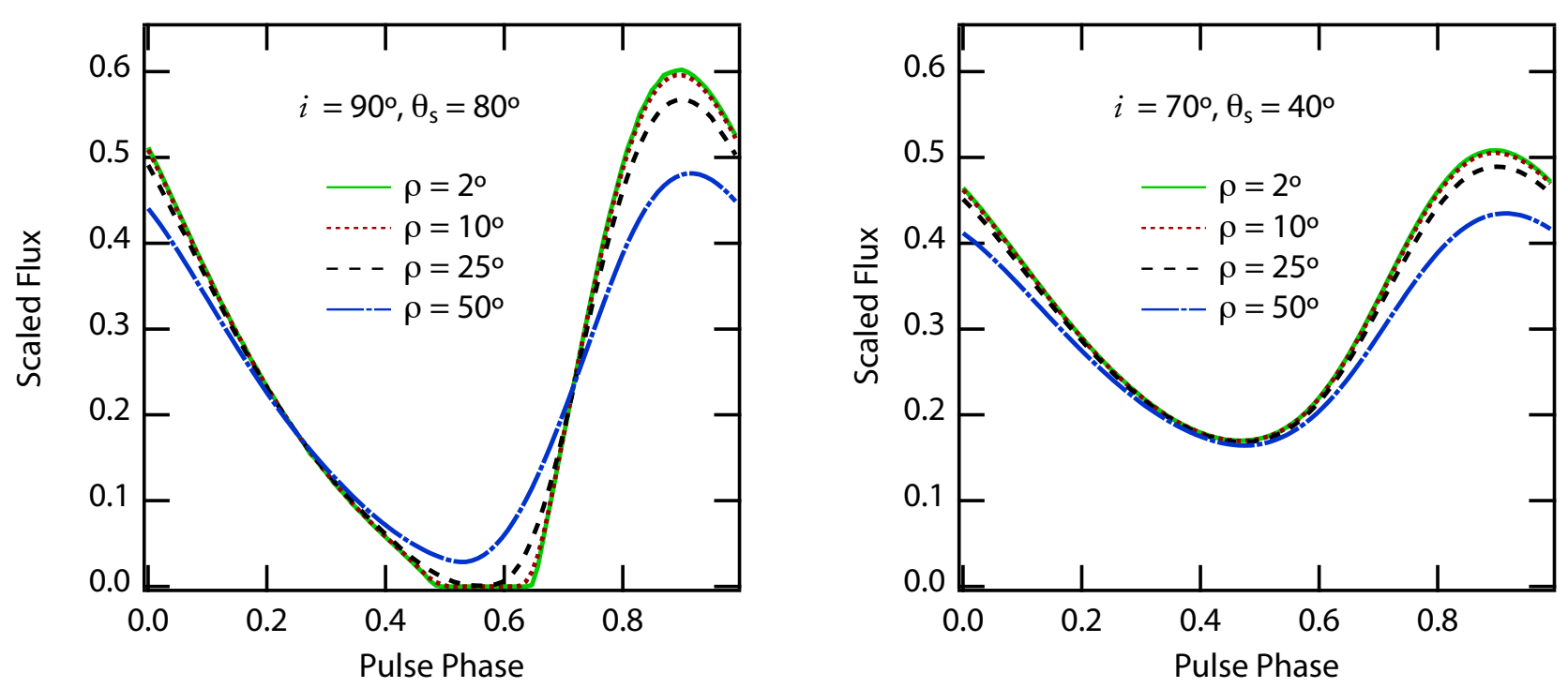

FIG. 1. - Example pulse profiles for two different spot configurations. In the left panel, the observer inclination is set to $i=90^{\circ}$ and the spot colatitude to $\theta_{s}=80^{\circ}$. In the right panel, they are set to $i=70^{\circ}$ and $\theta_{s}=40^{\circ}$. In both panels, the angular radius of the spot varies from $2^{\circ}$ to $50^{\circ}$. The flux has been scaled by $\left(50^{\circ} / \rho\right)^{2}$ in order to remove the primary dependence of the flux on the spot area. In all cases, we have set $M=1.6 \mathrm{M}_{\odot}, R=10 \mathrm{~km}$, and $f_{\mathrm{NS}}=600 \mathrm{~Hz}$.

der the assumption that the spot is infinitesimal in size. Lamb et al. (2009a, b; see also Psaltis et al. 2000) considered the effects of spot size on the amplitude of the pulse profile and found that the spot size has negligible effect as long as it is below $\sim 45^{\circ}$. However, they did not consider the effect of the spot size on the higher harmonics of the profile, which are necessary for measuring the stellar radius.

In this paper, we investigate the effect of spot size on pulse profiles for moderately spinning sources. Specifically, we determine the maximum spot size for which the spot can be considered small for the purpose of measuring the neutron-star radius. We then estimate the expected spot sizes as a function of spin frequency for rotationpowered and accretion-powered X-ray pulsars. In the case of X-ray burst oscillations, we find the fraction of the burst rise times during which a sufficient number of counts can be accumulated before the spot size becomes large enough to significantly bias the radius measurement from the pulse profile. We discuss the implications of our results for future missions that will observe pulse profiles, such as NICER and LOFT.

\section{PULSE PROFILES}

We use the ray-tracing code described in Bauböck et al. (2012) and Psaltis \& Özel (2014), which calculates pulse profiles using the Hartle-Thorne metric to approximate the spacetime around a moderately spinning neutron star. This metric is formally correct to second order in spin frequency and allows for the stellar surface to become oblate and the mass distribution to acquire an appropriate quadrupole moment. Our algorithm also accounts for frame dragging around the star, time of flight delays, and Doppler shifts and aberration due to the motion of the stellar surface.

The propagation of photons to infinity depends on seven parameters of the neutron star: the mass, the equatorial radius, the spin frequency, the moment of inertia, the oblateness, the quadrupole moment, and the inclina- tion of the observer's line of sight to the spin axis. Of these, the mass, spin frequency, and inclination are independent and vary between different sources. The radius, oblateness, moment of inertia, and quadrupole moment of the star are determined, for a given spin, by the equation of state of the matter in its interior. However, there exist empirical relations between several of these parameters that hold to high accuracy, for a range of equations of state (e.g., Bauböck et al. 2013; Yagi \& Yunes 2013). We use the relations in Bauböck et al. (2013) to calculate the oblateness, moment of inertia, and quadrupole moment for a star with a given mass, radius, and spin frequency.

By using the universal relations described above, we reduce the number of parameters for each pulse profile to six: the mass and radius of the neutron star, the spin frequency, the inclination of the observer to the spin axis, and the colatitude and angular radius of the hotspot. For the simulations presented in this work, we fix the mass at $1.6 \mathrm{M}_{\odot}$, the radius at $10 \mathrm{~km}$, and the spin frequency at $600 \mathrm{~Hz}$. We calculate pulse profiles over the full range of observer inclinations $i$ and spot colatitudes $\theta_{s}$. We define the observer's inclination as the angle between the the observer's line of sight and the spin axis, such that $i=0^{\circ}$ and $i=180^{\circ}$ correspond to an observer directly over the north and south pole of the neutron star, respectively, while $i=90^{\circ}$ corresponds to an observer in the equatorial plane. We vary the colatitude of the spot $\theta_{s}$, such that a spot on the pole has a colatitude $\theta_{s}=0^{\circ}$ and a spot on the equator corresponds to $\theta_{s}=90^{\circ}$.

Figure 1 shows several example pulse profiles for a variety of parameters. For all neutron-star configurations, the flux observed from the hotspot is proportional (to first order) to its area. In this figure, we have scaled the flux by this approximation to the spot area in order to highlight the more subtle changes in the profile shape introduced by increasing the spot size. In the left panel, the observer is in the equatorial plane of the neutron star, while the spot is near the equator. In this configuration, 


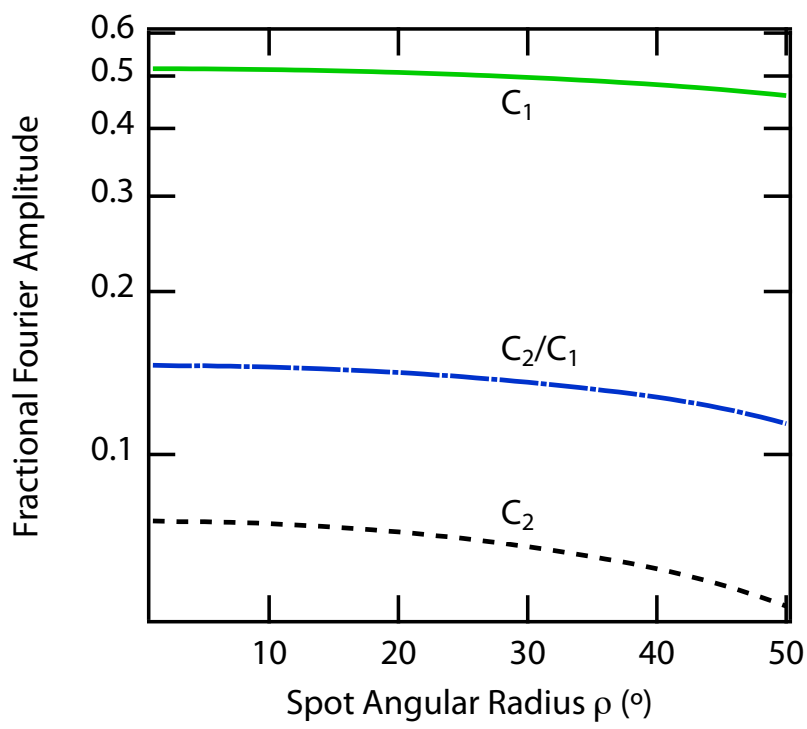

FIG. 2.- Fractional Fourier amplitudes of pulse profiles as a function of spot size. The observer inclination is $i=70^{\circ}$ and the spot colatitude is $\theta_{s}=40^{\circ}$. The remaining parameters are as in Figure 1 The solid and dotted lines show the first and second Fourier amplitudes, respectively. The dashed-dotted line shows the ratio between the two components.

the spot is eclipsed behind the neutron star for a fraction of the spin period. As the spot grows in size, the duration of the eclipse decreases until the spot is visible at all phases.

The right panel of Figure 1 shows profiles for a smaller observer inclination and spot colatitude. In this case, the spot is visible at all phases, regardless of its size. At larger angular radii, however, the amplitude of the profile still decreases. Moreover, the higher order harmonics also decrease, leading to a more symmetric and sinusoidal profile.

Since observations of neutron-star pulse profiles are usually limited by photon counts, information is often extracted by means of decomposing profiles into their Fourier components (Poutanen \& Beloborodov 2006; Psaltis et al. 2014). The number of parameters of the neutron star and the hotspot that can be constrained increases with the number of Fourier components that can be measured from the lightcurve. We limit our analysis to realistic situations where the fundamental and the second harmonics can be measured in at least two energy bands.

As expected from the examples in Figure 1, in addition to encoding information about the neutron star itself and the location of the hotspot, the Fourier amplitudes of the pulse profiles also depend on the size of the hotspot. In particular, as the size of the spot increases, the higher order harmonics are damped and the profile becomes more sinusoidal. In Figure 2, we show the amplitude of the first and second Fourier components, labeled $C_{1}$ and $C_{2}$ respectively, for a specific configuration of the observer inclination and the spot colatitude. For larger spot sizes, both the first and second harmonics decrease in amplitude. However, the second harmonic decreases more quickly than the first, causing the ratio between them to decrease also, as shown by the dashed-dotted line. This introduces a potential bias in the measurement of neutron-star radii, since the principal effect of a smaller radius is the reduction of the higher-order harmonics and, thereby, of the ratio $C_{2} / C_{1}$.

\section{BIASES IN RADIUS MEASUREMENTS}

We wish to determine the extent to which the common assumption of an infinitesimally small spot is justified for the purpose of measuring the radius of a neutron star from its pulse profile. In order to quantify the effect of the spot size on measurements of the radius, we calculate profiles with a very small spot (with an angular radius $\rho=2^{\circ}$ ) and quantitatively compare these to profiles with larger spots. We find the maximum spot size for which the assumption that the spot is infinitesimally small is reasonable, i.e., leading to a radius bias that is less than $10 \%$, allowing for meaningful constraints on the equation of state.

Using the relationships from Bauböck et al. (2013) for a neutron star of known spin frequency and the assumption that the hotspot is small, one can reduce the number of model parameters that need to be determined from the pulse profiles to four: the mass and radius of the neutron star, the colatitude of the hotspot, and the inclination of the observer. The number of observable quantities obtained from a pulse profile, on the other hand, depends to some extent on the relative inclinations of the observer and the spot to the spin axis of the neutron star. We will consider two cases: when the spot is visible for all phases, and when the spot becomes eclipsed behind the star for part of the spin period.

In the first case, we can measure the radius directly using the first two Fourier components of the pulse profiles at two broad energy ranges as the four required observables (see discussion in Psaltis et al. 2014). All four observables carry information about the radius of the neutron star because Doppler shifts and aberration, which are proportional to the stellar radius, affect their values. Following Psaltis et al. (2014), we will use the ratio of amplitudes of the first two Fourier harmonics, which to leading order is proportional to the Doppler shift (see below), in order to quantify the effect of the spot size on the radius measurement.

The second case arises when both the hotspot and the observer are located near the stellar equator. Although the strong lensing of photons increases the fraction of the surface area visible to a distant observer, there is a region which is hidden from sight in this configuration. When the spot is in this region, none of its flux reaches the observer. This eclipse introduces sharp edges to the pulse profile and causes many higher harmonics to become large. However, the amplitudes of these higher order harmonics are not independent, as they are caused by the sharp eclipse. In practice, the eclipse introduces one additional measurable parameter, which is its duration. For a given spot colatitude and observer's inclination, a spot with a smaller radius will tend to have shorter eclipses, as the lensing is stronger and a smaller fraction of the stellar surface is hidden. However, a large hotspot can mimic the effects of a smaller radius. As the spot grows, the fraction of the profile during which it is completely hidden from the observer decreases. In this second case, we will determine the effect of the spot size on the eclipse duration in order to quantify the possible bias in radius measurements.

In order to estimate the error introduced in the radius 


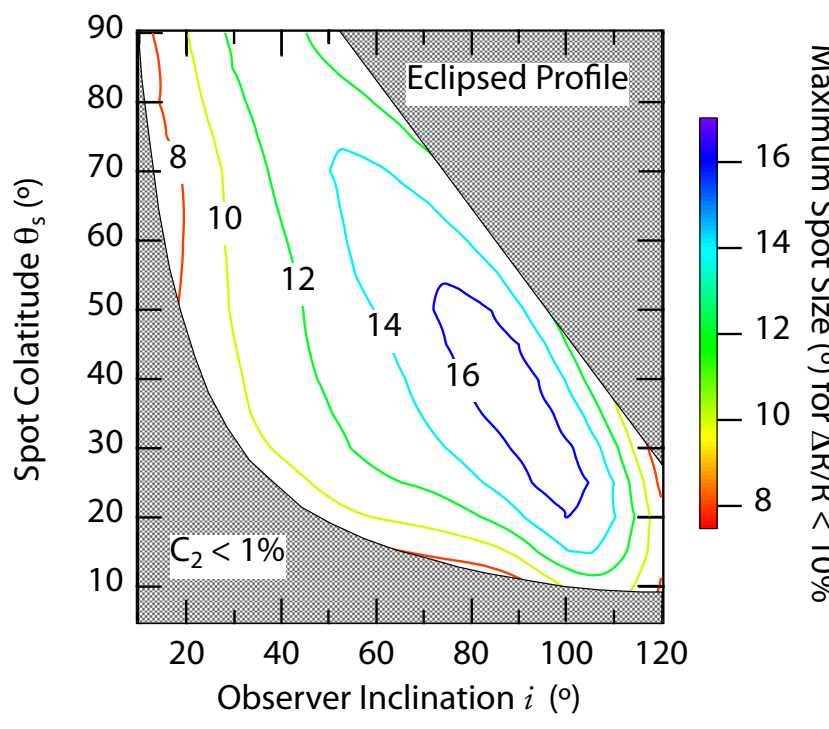

FIG. 3.- Maximum spot angular radius that leads to a $\leq 10 \%$ error in radius measurement as a function of the spot colatitude and observer inclination. If a radius measurement is made under the assumption of an infinitesimally small spot, the contours correspond to the spot size for which the measurement differs from the true neutron-star radius by ten percent.

measurement by a non-negligible spot size, we use the relation between the radius and the ratio of the first and second harmonics (Psaltis et al. 2014)

$$
\frac{C_{2}}{C_{1}} \approx \frac{4 \pi f}{c} R \sin i \sin \theta_{s} .
$$

This scaling was derived for a slowly spinning star in the Schwarzschild metric and is only expected to hold approximately for the most rapidly spinning stars. However, our purpose here is only to approximate the error in the radius measurement introduced by large spot sizes, for which this expression is sufficient.

Our goal is to calculate the maximum spot size at which the change introduced to the ratio $C_{2} / C_{1}$ due to the spot size is comparable to the difference introduced to this quantity by changing the radius by $10 \%$. To determine this maximum size, we first calculate the change in the ratio $C_{2} / C_{1}$ as

$$
\Delta\left(\frac{C_{2}}{C_{1}}\right) \approx 0.025\left(\frac{\Delta R}{1 \mathrm{~km}}\right)\left(\frac{f}{600 \mathrm{~Hz}}\right) \sin i \sin \theta_{s} .
$$

Next, we calculate the change in this ratio introduced by increasing the spot size. Specifically, for each pair of angles $i$ and $\theta_{s}$, we calculate a pulse profile and its Fourier components for a range of spot sizes between $2^{\circ}$ and $50^{\circ}$. As the spot grows in size, there is an increasing change in the ratio $C_{2} / C_{1}$. Comparing the two, we find the largest spot size $\rho$ for which this change is smaller than the error corresponding to a radius uncertainty of $10 \%$ given by equation 2 .

The contours in Figure 3 show the spot size which corresponds to a maximum error of $10 \%$ in radius. For most of the parameter space, we find that a spot size of up to $10^{\circ}-18^{\circ}$ introduces biases in the radius that are smaller than $10 \%$. The maximum allowable size generally increases with increasing inclination and decreasing spot colatitude. This effect is primarily due to the $\sin i \sin \theta_{s}$ term in equation (2). For small angles $i$ and $\theta_{s}$, the change in the ratio $C_{2} / C_{1}$ for a $10 \%$ change in the neutron-star radius becomes very small, leading to a small allowed spot size.

The contours in Figure 3 are calculated for a $10 \mathrm{~km}$ star. For stars with larger radii, the maximum allowed spot size that leads to a $10 \%$ bias in the radius measurement is smaller. This is due to the larger tangential velocity of the neutron star surface for a given spin frequency. As the linear velocity increases, the differential Doppler boost from the near and far edges of the spot reduces the amplitude of the second harmonic of the pulse profile more than for a smaller star at the same spin frequency. This leads to a larger bias in the radius measurement and therefore a smaller maximum spot size.

The Doppler boost scales as the tangential velocity squared and thus as $R^{2}$. Therefore, the spot size that introduces a fixed absolute bias in the radius measurement scales to first order as $1 / R^{2}$. In order to achieve a fractional accuracy of $10 \%$, the maximum spot size scales as $1 / R$.

The shaded parts of the parameter space in Figure 3 correspond to regions where our calculation of the maximum spot size is no longer valid or the measurement becomes unfeasible. In the lower region, corresponding to configurations where the observer and the spot are both near the rotational pole of the neutron star, the amplitude of the pulse profile is very small and the second harmonic is less than one percent. In the shaded region, the small amplitudes of pulse profiles make radius measurements of neutron stars unfeasible.

The upper shaded region in Figure 3 corresponds to the part of the parameter space where the spot is eclipsed for some fraction of the spin period. We focus on this region in Figure 4. The contours in the top panel of Figure 4 show the fraction of the profile during which the spot is eclipsed. Because of the strong lensing, the spot is hidden from view entirely for only a small portion of the parameter space, when the spot is near one pole and the observer's line of sight is near the other.

For the purpose of the top panel in Figure 4 , we have set the spot size to $\rho=2^{\circ}$. As the spot grows in size, however, it spends less time completely hidden behind the star and the eclipse duration decreases. If we were to make the assumption that the spot was infinitesimal in size, this decrease in the eclipse duration would introduce a bias in the parameters derived from the eclipse. In the lower panel of Figure 4, the contours correspond to the spot size that introduces an error equal to $10 \%$ of the total period to the eclipse duration. Note that the unevenness in the contours is a result of the numerical uncertainty in the eclipse duration. The dominant source of this uncertainty is the phase resolution of the pulse profile, which is on the order of $1 \%$. As in the case of no eclipses, spot sizes smaller than $15-20^{\circ}$ introduce marginal biases to the measurements of the eclipse duration and, hence, of the neutron-star radius.

\section{SOURCES}

We now compare our spot size limits to realistic situations where pulse profiles will be used to measure neutron-star radii with upcoming missions such as NICER or LOFT. We consider three types of sources in which X-ray pulsations have been observed. In the 


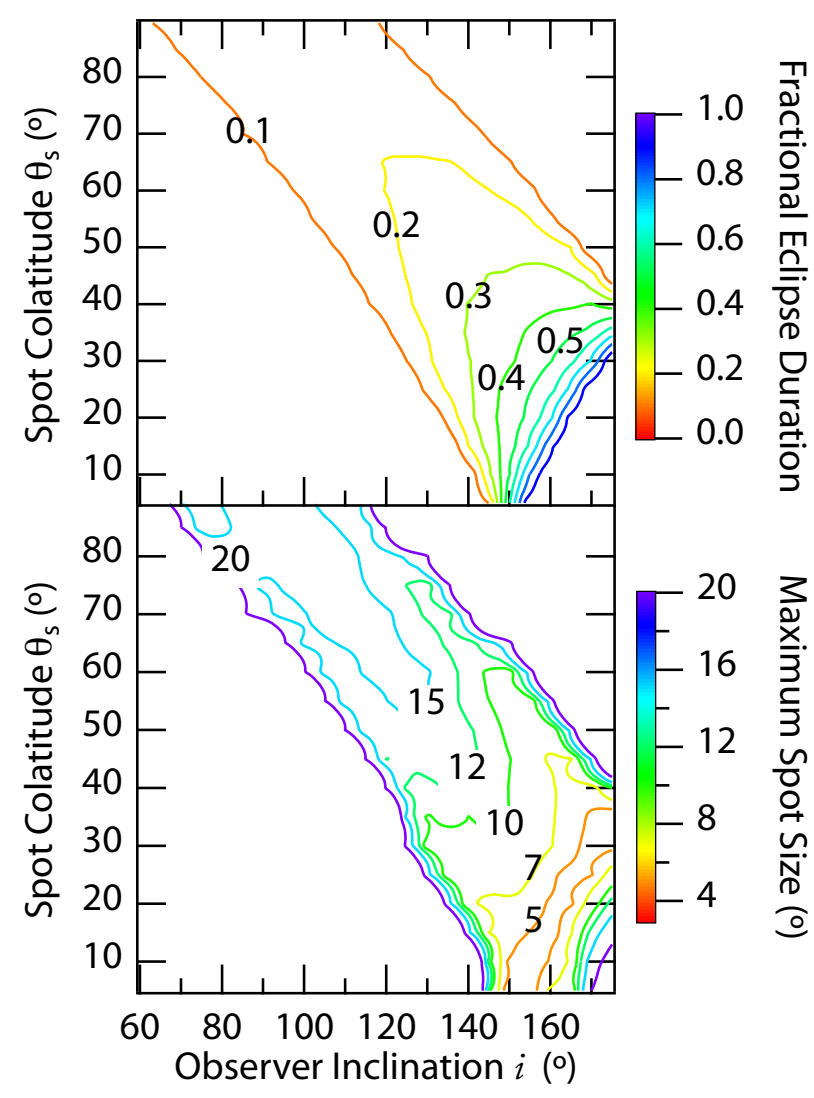

FIG. 4.- Region of the parameter space in which the spot is eclipsed for some fraction of the lightcurve. The upper panel shows the fractional duration of the eclipse for a $2^{\circ}$ spot angular radius: zero corresponds to a spot that is visible at all phases, while one corresponds to a spot that is never visible. For larger spots, the eclipse duration decreases. The lower panel shows the spot angular radius at which the duration of the eclipse is shorter compared to the eclipse for a $2^{\circ}$ spot by 0.1 of the period.

case of rotation-powered and accreting millisecond pulsars, the precise size of the spot is unknown a priori but can be estimated using physical arguments. For X-ray bursters, the spot grows during the rise of the burst until it covers the entire surface of the star. In each case, we estimate the size of the hotspot depending on the specific parameters of the system.

In rotation-powered pulsars, hotspots on the surface are generated by magnetic return currents. To obtain a size estimate, we consider the simple scenario in which the hotspot corresponds to the footprint of the open field lines on the stellar surface (Sturrock 1971). Open field lines are defined as those which pass through the light cylinder radius $R_{L C}$. This is the radius at which a cylinder corotating with the neutron star reaches the speed of light, i.e.,

$$
R_{L C} \equiv \frac{c}{2 \pi f_{\mathrm{NS}}},
$$

where $f_{\mathrm{NS}}$ is the spin frequency of the neutron star. In a dipole magnetic field, the quantity $\sin ^{2} \theta / r$, where $\theta$ is the polar angle, is constant along field lines, leading to

$$
\frac{\sin ^{2} \theta}{R}=\frac{\sin ^{2} \theta_{\mathrm{NS}}}{R_{\mathrm{NS}}}
$$

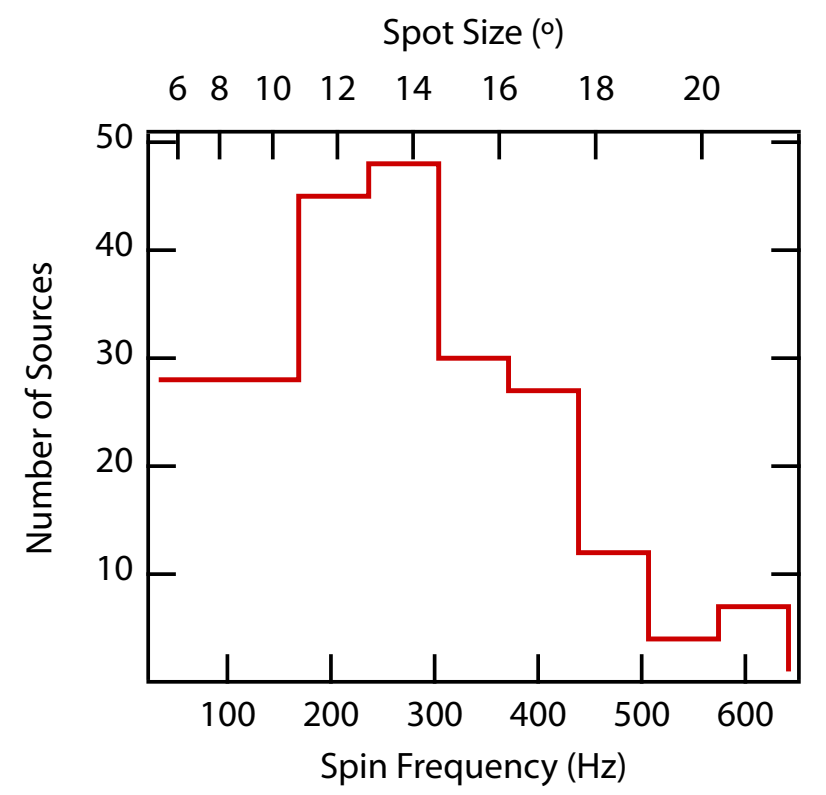

Fig. 5.- Histogram of the spin frequencies and the corresponding sizes of the polar caps of known rotation-powered millisecond pulsars. The lower axis shows the spin period, while the upper axis shows the spot size corresponding to equation (5).

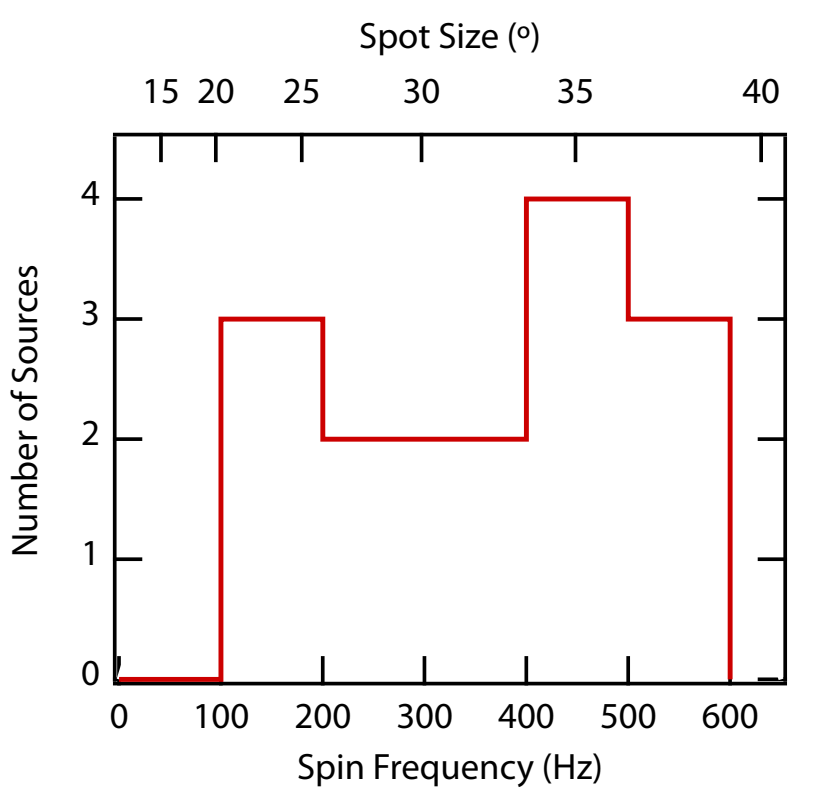

FIG. 6.- Histogram of accretion-powered X-ray pulsars from Watts (2012). As in Figure 5 the lower axis corresponds to the spin frequency, while the upper axis corresponds to the spot size derived from equation 7 . 


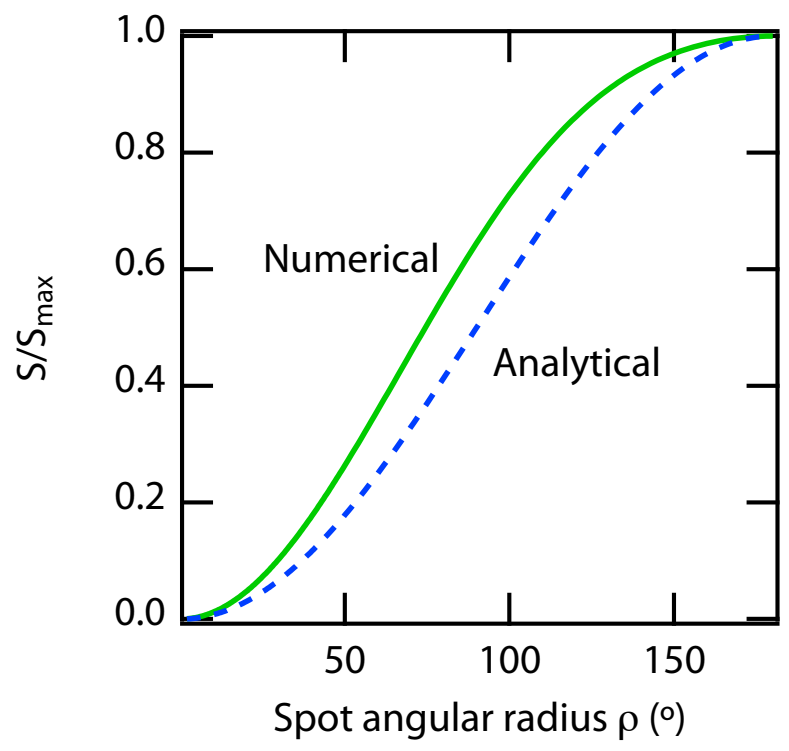

Fig. 7.- Apparent area of a hotspot averaged over the pulse profile as a function of the spot size. The analytical curve corresponds to equation (9). For the numerical curve, we have chosen a spot colatitude and observer inclination of $i=\theta_{s}=45^{\circ}$. For both curves, we have normalized the result by the apparent area of the whole neutron star such that $S\left(\rho=180^{\circ}\right)$ is equal to one.

The edge of the hotspot (where $\theta_{\mathrm{NS}}=\rho$ ) corresponds to the field line that reaches the light cylinder radius at the equator, where $\theta=90^{\circ}$. Therefore, we find the angular radius of the hotspot to be

$$
\rho_{\mathrm{RPP}}=\sin ^{-1}\left(\sqrt{\frac{2 \pi f_{\mathrm{NS}} R_{\mathrm{NS}}}{c}}\right) .
$$

Figure 5 shows the spin the distribution of rotationpowered X-ray pulsars from the online catalog compiled by D. Lorimer ${ }^{1}$. The lower axis shows the spin period of the pulsar, while the upper axis shows the spot size from equation (5), assuming a $10 \mathrm{~km}$ neutron star. The peak of the distribution is near $300 \mathrm{~Hz}$, corresponding to a spot size of $14^{\circ}$, and the majority of sources have spot sizes smaller than $18^{\circ}$. This implies that assuming that the hotspot on rotation-powered pulsars is infinitesimally small is a reasonable approximation for all but the most rapidly spinning sources.

In order to compare our analytical estimate with more detailed numerical simulations, we make use of the results of Bai \& Spitkovsky (2010a, b), who modeled the size and shape of the polar cap region under more realistic vacuum dipole and force-free magnetosphere conditions. They found that the spot size can vary slightly from the value derived in equation (5), especially for neutron stars in which the magnetic pole is misaligned from the spin axis. Moreover, they found that the spot is not circular in shape. For spots smaller than the limits we derived above, we expect the spot shape to be unimportant to the pulse profile. For larger spots, further investigation is needed to find the effect of non-circular spots on the shape of the pulse profiles.

In accreting sources, matter is funneled from the disk onto the stellar surface along magnetic field lines. We

\footnotetext{
${ }^{1}$ http://astro.phys.wvu.edu/GalacticMSPs/GalacticMSPs.txt
}

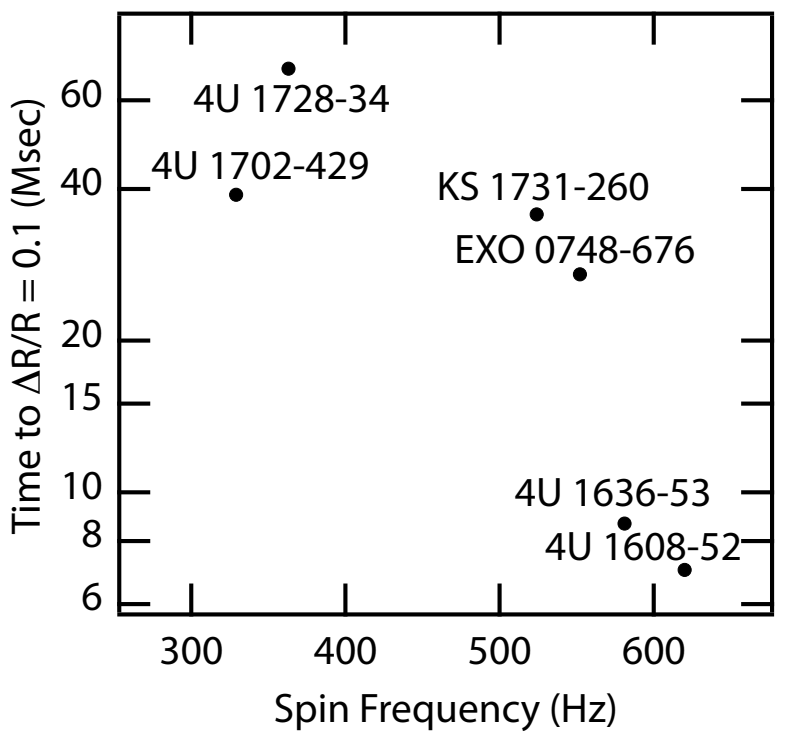

FIG. 8. - Time needed to achieve a $10 \%$ accuracy in the measurement of neutron-star radii using rise-time burst oscillations for several representative X-ray bursters, plotted against their spin frequencies.

will approximate the radius at which matter is transferred onto the magnetic field as the corotation radius $R_{c}$, i.e. the radius where a test mass in Keplerian orbit is corotating with the neutron star. In this case, it is straightforward to calculate the mass loading radius,

$$
\begin{aligned}
& R_{c} \equiv\left(\frac{G M}{4 \pi^{2} f_{\mathrm{NS}}^{2}}\right)^{1 / 3} \\
& \approx 2.46\left(\frac{R}{10 \mathrm{~km}}\right)^{-1}\left(\frac{M}{1.6 \mathrm{M}_{\odot}}\right)^{1 / 3}\left(\frac{f_{\mathrm{NS}}}{600 \mathrm{~Hz}}\right)^{-2 / 3}
\end{aligned}
$$

Using equation (4), we can again find the size of the hotspot as

$$
\rho_{\mathrm{AMXP}}=\sin ^{-1}\left(\sqrt{\frac{4 \pi^{2} R f_{\mathrm{NS}}}{G M}}\right) .
$$

In Figure 6, we show the histogram of the spin frequencies of accreting millisecond pulsars from Watts (2012). As in Figure 5, the lower axis shows the spin frequency, while the upper axis corresponds to the spot size derived from equation (7). Here, we assume a neutron-star radius of $10 \mathrm{~km}$ and a mass of $1.4 \mathrm{M}_{\odot}$ as before. As Figure 6 shows, the majority of accretion-powered pulsars have hotspots that are significantly larger than the maximum size we calculate above. Therefore, their pulse profiles will be significantly affected by the spot sizes and consequently by the spot shapes. Further study is needed to determine how to extract radius measurements from these sources.

The last class of sources we consider are X-ray bursters that show oscillations during the burst rise. In this case, the hotspot on the surface arises from the relatively slow spreading of the thermonuclear burning across the stellar surface. Therefore, during the rise, the spot size changes with time and the amplitude of pulsations decreases (see Strohmayer et al. 1996). We estimate here the fraction 
TABLE 1

Source Parameters

\begin{tabular}{|c|c|c|c|c|c|c|c|c|}
\hline Name & $\begin{array}{l}f_{\mathrm{NS}}{ }^{\mathrm{a}} \\
(\mathrm{Hz})\end{array}$ & $\begin{array}{c}A^{\mathrm{b}} \\
(\mathrm{km} / 10 \mathrm{kpc})^{2}\end{array}$ & $\begin{array}{l}t_{R}^{\mathrm{c}} \\
\mathrm{s}\end{array}$ & $\begin{array}{l}r_{b}{ }^{\mathrm{d}} \\
\mathrm{h}^{-1}\end{array}$ & $f_{o}{ }^{\mathrm{e}}$ & $N_{\text {osc }}{ }^{\mathrm{f}}$ & $N_{\text {bursts }} \mathrm{g}$ & $\begin{array}{l}t_{10 \%}{ }^{\mathrm{h}} \\
\text { Msec }\end{array}$ \\
\hline EXO 0748-646 & 552 & 114.0 & 4.9 & 0.24 & 0.02 & 38 & 1900 & 27.1 \\
\hline $4 \mathrm{U} 1608-52$ & 620 & 324.6 & 3.0 & 0.07 & 0.13 & 17 & 131 & 7.0 \\
\hline $4 \mathrm{U} 1636-53$ & 581 & 124.6 & 2.6 & 0.22 & 0.11 & 59 & 536 & 8.7 \\
\hline $4 \mathrm{U} 1702-429$ & 329 & 164.6 & 1.0 & 0.13 & 0.26 & 359 & 1381 & 39.0 \\
\hline $4 \mathrm{U} 1728-34$ & 363 & 121.6 & 1.0 & 0.20 & 0.10 & 400 & 4000 & 69.3 \\
\hline KS 1731-260 & 524 & 88.4 & 1.1 & 0.20 & 0.37 & 73 & 197 & 35.6 \\
\hline
\end{tabular}

a Spin frequency

b Blackbody normalization

c Average rise time

d Burst rate

e Fraction of bursts with oscillations in the rise

${ }^{\mathrm{f}}$ Number of bursts with oscillations needed to constrain $R_{\mathrm{NS}}$ to within $10 \%$

g Total number of bursts needed to constrain $R_{\mathrm{NS}}$ to within $10 \%$

h Observing time needed to constrain $R_{\mathrm{NS}}$ to within $10 \%$

of the burst oscillations for which we can assume that the spot is infinitesimal without introducing a large bias to the radius measurement.

Assuming that the spot size increases linearly during the burst rise from a point at $t=0$ to a spot covering the entire surface $\left(\rho=180^{\circ}\right)$ at the burst maximum, we write the angular radius as a function of time,

$$
\rho(t)=\frac{\pi t}{t_{R}},
$$

where $t_{R}$ is the rise time of the burst. This equation is formally valid only when the photon diffusion time from the burning layer to the photosphere is smaller than the lateral propagation time of the burning front such that the rise time of the burst is dominated by the lateral spreading. Nevertheless, for the purposes of our estimates here, we will use this approximation to calculate the time before the spot reaches some maximum size $\rho_{\max }$.

In the Newtonian case, we could calculate the emitting area on the stellar surface analytically,

$$
S_{\mathrm{spot}}^{\mathrm{Newton}}=\int_{0}^{\rho} 2 \pi R_{\mathrm{NS}}^{2} \sin \rho^{\prime} d \rho^{\prime}=2 \pi R_{\mathrm{NS}}^{2}(1-\cos \rho) .
$$

However, this does not take into account the lensing of the spot, which distorts its size and shape and increases the fraction of the stellar surface that is visible to a distant observer or the effects of phase averaging. We numerically calculate the average emitting area of the spot over all phases as in Psaltis et al. (2000). Figure 7 shows the emitting area as a function of the spot size for a configuration where the spot colatitude and the inclination to the observer are both $45^{\circ}$. The analytic expression derived in equation (9) is shown in the dashed line. As expected in this configuration, the analytic approximation underestimates the average flux, since gravitational lensing tends to increase the apparent surface area.

Converting this surface area into a countrate depends on the specifics of the spectrum emitted from the hotspot and the detector used. Since we are primarily interested in an approximate estimate of the time needed to constrain the radius, we assume that the hotspot emits a blackbody spectrum at a temperature of $2 \mathrm{keV}$. The detected flux then depends only on the angular size of the source, which we encode in the blackbody normalization $A$, in units of $(\mathrm{km} / 10 \mathrm{kpc})^{2}$. The countrate also depends on the detector efficiency, which we encode in the quantity $C$ that measures the number of photons detected from a $2 \mathrm{keV}$ blackbody with an angular size of $(1 \mathrm{~km} / 10 \mathrm{kpc})^{2}$. Then the countrate that will be observed for a spot of a given size becomes

$$
\frac{\text { counts }}{\text { second }}=\frac{S_{\mathrm{spot}}}{S_{\max }} A C,
$$

where $S_{\max }$ is the apparent area of the whole star. We find the total number of counts for a given burst by numerically integrating equation 10 from 0 to $\rho_{\max }$. In order to estimate total number of photons $N$ for a typical spot location and observer inclination, we set $\rho_{\max }$ to $15^{\circ}$ and $\mathrm{C}$ to 2700 counts s$^{-1}(10 \mathrm{kpc} / \mathrm{km})^{2}$ (for the LOFT Large Area Detector, T. Güver 2015, private communication) and find

$$
N=2.64 A t_{R} .
$$

The number of counts necessary to measure the radius to $10 \%$ depends on the magnitude of the Fourier components as well as the geometry of the hotspot and the spin frequency of the neutron star. Psaltis et al. (2014) found that the number of counts necessary to constrain the radius to $10 \%$ is approximately

$$
\begin{aligned}
N \approx 4.7 \cdot 10^{4}\left(\frac{C_{1}}{0.38}\right)^{2}\left(\frac{f_{\mathrm{NS}}}{600}\right)^{2}\left(\frac{R_{\mathrm{NS}}}{10 \mathrm{~km}}\right)^{2} & \left(\frac{\sin i}{0.71}\right)^{2}\left(\frac{\sin \theta_{s}}{0.71}\right)^{2},
\end{aligned}
$$

which is consistent with numerical simulations by Lo et al. (2013). Here, we have set $i=\theta_{s}=45^{\circ}$ as above. For this configuration, we also find the first Fourier harmonic $C_{1}$ to have a fractional amplitude of 0.38 for a spot size of $\rho=2^{\circ}$.

We now combine equation (11) with equation (12) to find the number of individual bursts which must be added to measure the radius to a $10 \%$ accuracy. If we further know the burst rate, $r_{b}$, and the fraction of bursts showing oscillations during their rise, $f_{o}$, we can calculate the 
observing time necessary for each source as

$$
t_{10 \%}=\frac{1.79 \cdot 10^{4}}{A t_{R}} r_{b} f_{o} .
$$

We present in Table 1 the parameters for several bursting sources for which oscillations were detected during the rise time with the /textitRossi X-ray Timing Explorer $(R X T E)$. Here, we use the burst rise times, the burst frequency, and the fraction of bursts with oscillations from Galloway et al. (2008). We use the blackbody normalization $A$ from Güver et al. (2012) for sources $4 \mathrm{U}$ 1728-34, 4U 1702-429, KS 1731-260, and 4U 163653, from Özel (2006) for EXO 0748-676, and from Güver et al. (2010) for 4U 1608-52. We have chosen only the sources for which the spin frequency and blackbody normalization are known. In Figure 8, we show the time needed to reach an accuracy of $10 \%$ in measuring the radius versus the spin frequency for these sources.

It is evident from Figure 8 that sources with higher spin rates in general allow for better constraints to the radius. This is because a faster spin leads to stronger Doppler effects, causing the pulse profile to become more asymmetric. This results in an increase in the higher Fourier harmonics and thereby an increase in the accuracy of the radius measurement.

In the preceding estimate, we used the fraction of bursts with rise-time oscillations as inferred from $R X T E$ observations. Using a future timing instrument with a larger collecting area such as LOFT will allow searching for burst oscillations during smaller time segments in the rise of the bursts. This increased sensitivity will most likely reveal oscillations in bursts in which the spreading time is short compared to the typical duration of the $R X T E$ time segments or the location of the ignition of the bursts gives rise to smaller amplitude oscillations. Those bursts, however, will not add an appreciable number of counts to the integrated pulse profiles that will be used for measuring neutron star radii.

\section{CONCLUSIONS}

In this paper, we focused on the problem of deriving neutron star parameters by modeling pulse profiles from hotspots on the stellar surface. In particular, we addressed an often made assumption that the spot size can be treated to be infinitesimal in size and investigated the conditions under which this leads to errors that can be tolerated. We calculated the maximum size for which the hotspot can be considered to be infinitesimal, both for derivations of the radius from the Fourier components of the profile and for profiles which include an eclipse.

We found that hotspots with angular radius smaller than $10^{\circ}-18^{\circ}$ produce profiles that are not significantly different from those with very small hotspots. The maximum allowed spot size that corresponds to a $10 \%$ error in the derived radius depends on both the location of the spot and the inclination of the neutron star spin axis to the observer's line of sight. In general, increasing the inclination and decreasing the colatitude lead to a larger allowed spot sizes.

Similarly, we found that the duration of eclipses is relatively insensitive to the size of the hotspot if it is below $10^{\circ}-20^{\circ}$. In this case, increasing observer inclination and decreasing spot colatitude lead to a smaller maximum spot size. Nevertheless, for some extreme regions of the parameter space, even a spot smaller than $5^{\circ}$ can have an eclipse duration that is significantly smaller than that of an infinitesimal spot.

Finally, we considered the implications of spot size limits for the upcoming NICER and the planned LOFT missions. We showed that, for rotation-powered millisecond X-ray pulsars, the majority of sources are expected to have spot sizes smaller than the limits derived above. For accretion-powered pulsars, we expect only the most slowly spinning sources to have hotspots small enough that the spot size can be neglected. For X-ray bursters, we calculated the fraction of the burst rise-time for which the spot is small enough to constrain the radius to within $10 \%$. We found that, for two sources (4U 1636-53 and $4 \mathrm{U} 1608-52$ ), it is possible to measure the radius to a $10 \%$ accuracy within a time of 7-9 Ms. Other sources with less optimal configurations require longer observations of 20-40 Ms in order to accurately measure their radii.

This research was conducted at the University of Arizona and is supported by NSF grants AST 1108753 and AST 1312034 as well as the NASA ADP award NNX12AE10G. All ray-tracing calculations were performed on the El Gato cluster that is funded by NSF grant 1228509 .

\section{REFERENCES}

Arons, J. 1981, ApJ, 248, 1099

Bai, X., \& Spitkovsky, A. 2010a, ApJ, 715, 1270

Bai, X., \& Spitkovsky, A. 2010b, ApJ, 715, 1282

Bauböck, M., Berti, E., Psaltis, D., \& Özel, F. 2013, ApJ, 777, 68

Bauböck, M., Psaltis, D., Özel, F., \& Johannsen, T. 2012, ApJ, 753,175

Bildsten, L. 1995, ApJ, 438, 852

Bogdanov, S., Rybicki, G., \& Grindlay, J. 2007, ApJ, 670, 668

Braje, T., Romani, R., \& Rauch, K. 2000, ApJ, 531, 447

Cadeau, C., Morsink, S., Leahy, D., \& Campbell, S. 2007, ApJ, 654,458

Frank, J., King, A., \& Raine, D. 2002, Accretion Power in Astrophysics, (3rd ed.; Cambridge: Cambridge Univ. Press)

Galloway, D., Muno, M., Hartman, J., Psaltis, D., \&

Chakrabarty, D. 2008, ApJS, 179, 360

Güver, T., Özel, F., Cabrera-Lavers, A., \& Wroblewski, P. 2010 ApJ, 712, 964

Güver, T., Psaltis, D., \& Özel, F. 2012, ApJ, 747, 76
Harding, A., \& Muslimov, A. 2001, ApJ, 556, 987

Harding, A., \& Muslimov, A. 2002, ApJ, 568, 862

Lamb, F., Boutloukos, S., Van Wassenhove, S., et al. 2009a, ApJ, 706,417

Lamb, F., Boutloukos, S., Van Wassenhove, S., Chamberlain, R., Lo, K.H., \& Miller, M. 2009b, ApJ, 705, 36

Lattimer, J., \& Prakash, M. 2001, ApJ, 550, 426

Lindblom, L. 1992, ApJ, 398, 569

Lo, K.H., Miller, M., Bhattacharyya, S., \& Lamb, F. 2013, ApJ, 776,19

Miller, M., \& Lamb, F. 1998, ApJ, 499, 37

Morsink, S., Leahy, D., Cadeau, C., \& Braga, J. 2007, ApJ, 663, 1244

Muno, M. P., Özel, F., \& Chakrabarty, D. 2002, ApJ, 581, 550

Muno, M. P., Özel, F., \& Chakrabarty, D. 2003, ApJ, 595, 1066

Özel, F. 2006, Nature, 441, 1115

Özel, F., \& Psaltis, D. 2009, Phys. Rev. D, 80, 103003

Poutanen, J., \& Beloborodov, A. 2006, MNRAS, 373, 836 
Psaltis, D. 2008, Phys. Rev. D, 77, 6

Psaltis, D., Özel, F., \& DeDeo, S. 2000, ApJ, 544, 390

Psaltis, D. \& Özel, F. 2014, ApJ, 792, 87

Psaltis, D., Özel, F., \& Chakrabarty, D. 2014, ApJ, 787, 136

Read, J. Lackey, B., Owen, B., \& Friedman, J. 2009,

Phys. Rev. D, 79, 124032

Ruderman, M., \& Sutherland, P. 1975, ApJ, 196, 51

Schoelkopf, R., \& Kelley, R. 1991, ApJ, 375, 696
Strohmayer, T. 1992, ApJ, 388, 138

Strohmayer, T., Zhang, W., Swank, J., et al. \& Lee, U. 1996, ApJ, 469, 9

Sturrock, P. 1971, ApJ, 164, 529

Watts, A. 2012, ARA\&A, 50, 609

Yagi, K., \& Yunes, N. 2013, Phys. Rev. D, 88, 023009 